\title{
S100B promotes chemoresistance in ovarian cancer stem cells by regulating p53
}

\author{
TAO YANG ${ }^{1 *}$, JIANAN CHENG $^{2 *}$, JUNHAO YOU $^{1 *}$, BING YAN $^{1}$, HUI LIU $^{1}$ and FANG LI \\ ${ }^{1}$ Department of Oncology, Hainan Branch of Chinese PLA General Hospital, Sanya, Hainan 572013; \\ ${ }^{2}$ Institute of Cancer, Xinqiao Hospital, Third Military Medical University, Chongqing 400037, P.R. China
}

Received December 6, 2017; Accepted June 8, 2018

DOI: $10.3892 / o r .2018 .6527$

\begin{abstract}
Chemoresistance is one of the most important causes of ovarian cancer-related deaths. Recently, cancer stem cells (CSCs) have been recognized as the source of chemoresistance in ovarian cancer. However, the underlying mechanisms that regulate the chemoresistance of ovarian CSCs (OCSCs) remain unclear. The aim of the present study was to investigate the roles of S100B in the regulation of OCSC chemoresistance, which provides a novel therapeutic target. We observed high expression of S100B in CD133+ OCSCs derived from ovarian cancer cell lines and primary tumors and in cisplatin-resistant patient samples. Then, we determined that S100B knockdown promoted the apoptosis of OCSCs after treatment with different concentrations of cisplatin. The underlying mechanism of S100B-mediated chemoresistance in OCSCs may be through p53 inhibition. Furthermore, drug-resistance genes, including MDR1 and MRP1, were involved in the process of S100B-mediated OCSC chemoresistance. In conclusion, our results elucidated the importance of $\mathrm{S} 100 \mathrm{~B}$ in the maintenance of OCSC chemoresistance, which may provide a promising therapeutic target for ovarian cancer.
\end{abstract}

\section{Introduction}

Ovarian cancer is the most lethal cancer among gynecological malignancies (1). As the first-line treatment for ovarian cancer

Correspondence to: Dr Hui Liu or Dr Fang Li, Department of Oncology, Hainan Branch of Chinese PLA General Hospital, Jianglin Street, Haitang, Sanya, Hainan 572013, P.R. China

E-mail: woshiliuhui1995@aliyun.com

E-mail: 422252147@qq.com

*Contributed equally

Abbreviations: OCSCs, ovarian cancer stem cells; EMT, epithelial-mesenchymal transition; shRNA, short hairpin RNA; MDR1, multidrug resistance gene 1; MRP1, multidrug resistance-associated protein 1; NSCLC, non-small cell lung carcinoma; RAGE, receptor for advanced glycation end products; MDM2, mouse double minute 2 homolog gene

Key words: S100B, OCSCs, chemoresistance, p53 patients, cisplatin-centered chemotherapy has some benefits but is not sufficient (2). The major cause of treatment failure may be the resistance to chemotherapy of certain ovarian cancer cells, which are called ovarian cancer stem cells (OCSCs) (3). OCSCs are a minority population of cancer cells in ovarian cancer, and they harbor many properties of stem cells, such as self-renewal, differentiation and tumorigenicity. In past decades, numerous lines of evidence have revealed the key roles of OCSCs in the chemoresistance of ovarian cancer (4). However, the underlying mechanisms have not been extensively investigated. Thus, elucidating the chemoresistance mechanisms in OCSCs may be important for improving patient prognoses.

$\mathrm{S} 100 \mathrm{~B}$ is a type of $\mathrm{EF}$-hand $\mathrm{Ca}^{2+}$ binding protein that is aberrantly expressed in many types of cancers, including melanoma, colon and ovarian cancer (5). It has been reported that S100B mediates the resistance of melanoma cells to UV radiation (6). Moreover, in another study, serum S100B levels could be used for the prediction and monitoring of responses to chemoimmunotherapy in metastatic malignant melanoma (7). These results revealed that $\mathrm{S} 100 \mathrm{~B}$ may be involved in the resistance of melanoma to various treatments. In our previous study, we reported that $\mathrm{S} 100 \mathrm{~B}$ was highly expressed in OCSCs, and promoted self-renewal and tumorigenicity in those cells (8). However, whether S100B can also mediate the chemoresistance of OCSCs is not clear.

Accumulating evidence has shown that S100B inactivates p53 to interfere with the proliferation, apoptosis and EMT of tumor cells $(6,9,10)$. We have also reported that $\mathrm{S} 100 \mathrm{~B}$ can mediate the stemness of OCSCs by regulating p53 (8). As a well-known tumor suppressor, p53 plays an important role in the chemoresistance of ovarian cancer $(11,12)$. Thus, we hypothesized that S100B may promote the chemoresistance of OCSCs by regulating $\mathrm{p} 53$.

Herein, we reported that $\mathrm{S} 100 \mathrm{~B}$ was highly expressed in OCSCs and cisplatin-resistant ovarian cancer samples. S100B knockdown significantly sensitized OCSCs to cisplatin treatment. Mechanistically, S100B promoted OCSC resistance to cisplatin by upregulating MDR1 and MRP1 via p53 inactivation.

\section{Materials and methods}

Patient samples and cell lines. The human ovarian cancer cell line A2780 was obtained from the American Type Culture 
Collection (ATCC, Manassas, VA, USA) and the authenticity was recently tested. A2780 cells were cultured in Dulbecco's modified Eagle's medium (DMEM)/H (HyClone Laboratories; GE Healthcare Life Sciences, Logan, UT, USA) with $10 \%$ fetal bovine serum (FBS; Gibco; Thermo Fisher Scientific, Inc., Waltham, MA, USA) and $1 \%$ penicillin/streptomycin (Beyotime Institute of Biotechnology, Shanghai, China). The primary tumor tissue mentioned in the present study was from surgical resection of one patient (female, 56 years old) categorized with stage III serous ovarian cancer in November 2017 in Hainan Branch of Chinese PLA General Hospital. The present study was approved by the Institutional Review Board of the Hainan Branch of Chinese PLA General Hospital, and informed consent was obtained from this patient.

Generation and culture of OCSCs. The CD133+ OCSCs from the A2780 cell line were isolated by serum-free culture, as previously described (13). Briefly, after dissociation with trypsin (Invitrogen; Thermo Fisher Scientific, Inc., Waltham, MA, USA), single A2780 cells were cultured in low-attachment plates under stem cell conditions. The $\mathrm{CD}_{133^{+}}$OCSCs from patient samples were isolated by magnetic bead sorting (MACS) using the MACS system, as previously described (13). Briefly, single-cell suspensions were obtained by enzymatic dissociation of primary tumor samples, incubated with microbeads conjugated to anti-CD133/1, and sorted with a MACS column (both from Miltenyi Biotec GmbH, Bergisch Gladbach, Germany). The sorted OCSCs were maintained in serum-free DMEM/F12 (HyClone Laboratories; GE Healthcare Life Sciences) supplemented with basic fibroblast growth factor (10 ng/ml), epithelial growth factor (20 ng/ml) (both from PeproTech, Inc., Rocky Hill, NJ, USA) and recombinant insulin $(5 \mu \mathrm{g} / \mathrm{ml}$; Sigma-Aldrich; Merck KGaA, Darmstadt, Germany). The sorted CD133 cells and the A2780 cells were cultured in DMEM/H (HyClone Laboratories; GE Healthcare Life Sciences) supplemented with 10\% FBS (Gibco; Thermo Fisher Scientific, Inc.) and 1\% penicillin/streptomycin (Beyotime Institute of Biotechnology).

GEO database. These two patient cohorts involved in this study were derived from the GEO database in the NCBI website. The first patient cohort in Fig. 2D was obtained from GSE51373 (14), which compared the differences of gene expression between 13 patients sensitive to cisplatin and 10 patients resistant to cisplatin with stage III-IV high-grade serous ovarian cancer (https://www.ncbi.nlm.nih.gov/geo/query/acc. cgi?acc=GSE51373). In addition, the other patient cohort in Fig. 5C was obtained from GSE13876 (15), which consisted of gene expression data from 157 patients with advanced-stage serous ovarian cancer; however, the control was not described in this study (https:/www.ncbi.nlm.nih.gov/geo/query/acc. cgi?acc=GSE13876). All of the patients in the two databases mentioned in this study were diagnosed as advanced-stage serous ovarian cancer (III-IV stage), which was demonstrated by key clinical characteristics, histological type and stage. In addition, other clinical characteristics, such as age, may not be associated with gene expression and cisplatin efficacy in ovarian cancer (16).
$C C K-8$ assay. A total of $5 \times 10^{3}$ OCSCs were treated to obtain single cells and seeded into 96 -well plates. Then, the OCSCs were treated with different concentrations $(0,10$ or $20 \mu \mathrm{M}$ ) of cisplatin. After $48 \mathrm{~h}$, the assay was performed by adding $10 \mu \mathrm{l}$ of Cell Counting Kit-8 (CCK-8) solution (Beyotime Institute of Biotechnology) into each well, incubating the samples for $4 \mathrm{~h}$ and measuring the absorbance at $450 \mathrm{~nm}$. The cell viability was calculated as follows: Cell viability $=\left(\mathrm{Ab}_{\text {exp }}-\mathrm{Ab}_{\text {blank }}\right) /\left(\mathrm{Ab}_{\text {con }}-\mathrm{Ab}_{\text {blank }}\right) \times 100 \%$.

Immunofluorescence staining. Digested A2780 cells or spheres of OCSCs were attached to coverslips or slides, respectively. After being fixed with $4 \%$ paraformaldehyde solution (Beyotime Institute of Biotechnology) for $30 \mathrm{~min}$, the samples were blocked with 5\% BSA (Beyotime Institute of Biotechnology) for $20 \mathrm{~min}$ at room temperature and incubated with mouse anti-S100B (1:200; cat. no. ab218513; Abcam, Cambridge, MA, USA) overnight at $4^{\circ} \mathrm{C}$. After being washed with PBS, the samples were incubated with FITC-conjugated (1:200; cat. no. A0568; Beyotime Institute of Biotechnology) for $30 \mathrm{~min}$ at $37^{\circ} \mathrm{C}$. After washing, the nuclei were stained with DAPI (Beyotime Institute of Biotechnology). Finally, the samples were mounted and then viewed under an Olympus confocal laser scanning microscope (Olympus FV3000; Olympus Corp., Tokyo, Japan).

Real-time PCR. Total RNA was isolated from OCSCs and non-OCSCs using TRIzol and the PureLink total RNA purification kit (Invitrogen; Thermo Fisher Scientific, Inc.) according to the manufacturer's instructions. Then, reverse transcription and quantitative real-time PCR were performed with a cDNA Reverse Transcription kit and a SYBR Kit (both from Takara Biotechnology Co., Ltd., Dalian, China), respectively. The PCR amplification was performed with 40 cycles with the following protocol: Denaturation at $94^{\circ} \mathrm{C}$ for $30 \mathrm{sec}$, annealing at $55^{\circ} \mathrm{C}$ for $30 \mathrm{sec}$, and lastly extension at $72^{\circ} \mathrm{C}$ for $1 \mathrm{~min}$. The relative gene expression was calculated with the $\mathrm{Cq}$ value (17), and $\beta$-actin was the reference gene. The primers used herein were as follows: S100B, 5'-AGCTGGAGAAGGCCATGG TG-3' (forward), and 5'-GAACTCGTGGCAGGCAGTAG-3' (reverse); MDR-1, 5'-GCCTGGCAGCTGGAAGACAAATAC ACAAAATT-3' (forward), and 5'-CAGACAGCAGCTGAC AGTCCAAGAACAGGACT-3' (reverse); MRP-1, 5'-CCTGCA GCAGAGAGGTCTTTTC-3' (forward) and 5'-GGCATATAG GCCCTGCAGTTC-3' (reverse); and $\beta$-actin, 5'-TGGAAT CCTGTGGCATCCATGAAAC-3' (forward) and 5'-TAAAAC GCAGCTCAGTAACAGTCCG-3' (reverse).

Western blotting. Total protein was extracted with RIPA buffer supplemented with $1 \%$ phenylmethanesulfonyl fluoride (PMSF), and then a BCA kit (both from Beyotime Institute of Biotechnology) was used to examine the concentrations. For western blotting, $40 \mu \mathrm{g}$ of protein were separated by $12 \%$ SDS-PAGE and transferred onto polyvinylidene difluoride (PVDF) membranes. After blocking with 5\% BSA (Beyotime Institute of Biotechnology) for $20 \mathrm{~min}$ at room temperature, the following primary antibodies were used to incubate the PVDF membranes overnight at $4^{\circ} \mathrm{C}$ : mouse anti-S100B (1:200; cat. no. ab218513; Abcam), mouse anti- $\beta$-actin (1:500; cat. no. AA128; Beyotime Institute of Biotechnology), rabbit 
A

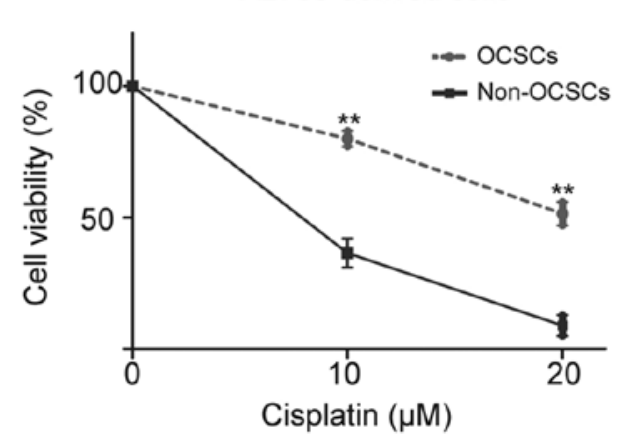

B

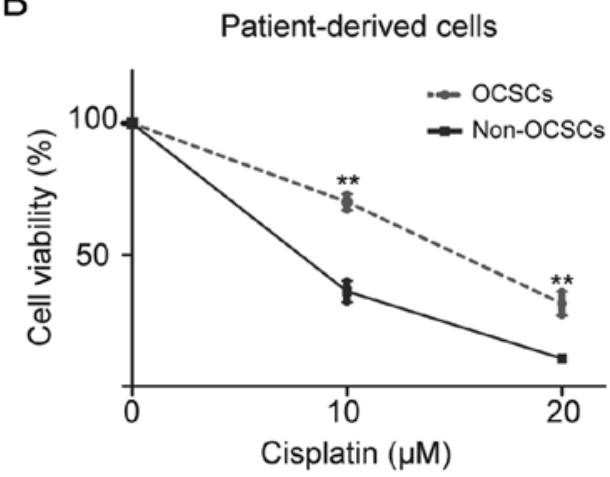

Figure 1. OCSCs are more resistant to cisplatin than non-OCSCs. (A) A2780-derived OCSCs and non-OCSCs were treated with different concentrations of cisplatin, and their viability was assessed by a CCK-8 assay. (B) Patient-derived OCSCs and non-OCSCs were treated with different concentrations of cisplatin, and their viability was assessed by a CCK- 8 assay. ${ }^{* *} \mathrm{P}<0.01$. OCSCs, ovarian cancer stem cells.

anti-p53 (1:500; cat. no. 10442-1-AP; ProteinTech Group, Inc., Chicago, IL, USA), rabbit anti-p-p53 (1:500; cat. no. 9284; Cell Signaling Technology,Inc., Danvers, MA,USA), rabbit-anti-p21 (1:500; cat. no. ab109199) and rabbit-anti-MDM2 (1:500; cat. no. ab38618; both from Abcam). After washing with $0.1 \%$ TBS-Tween-20 (TBST) (1 ml Triton X-80 in $11 \mathrm{TBS}$ solution), a goat anti-mouse secondary antibody (1:5,000; cat. no. A0216) and a goat anti-rabbit secondary antibody (1:5,000; cat. no. A0239; both from Beyotime Institute of Biotechnology) were used to incubate the PVDF membranes for $30 \mathrm{~min}$ at room temperature. Then, the images were detected with an enhanced chemiluminescence (ECL) kit (Thermo Fisher Scientific, Inc.) using the Chemilmanger ${ }^{\mathrm{TM}}$ 5500 system (Alpha Innotech Corporation, San Leandro, CA, USA). The gray values were calculated using ImageJ software (http://rsb.info.nih.gov//), and $\beta$-actin was used as the loading control for normalization.

Lentivirus transfection. S100B-shRNA and NT-shRNA lentiviruses were commercially obtained (Shanghai GeneChem Co., Ltd., Shanghai, China). Briefly, a stem-loop structure oligonucleotide containing S100B or p53-target sequences was cloned under the control of the human U6 promoter in lentiviral vectors, which also contained a GFP reporter. The sequences were as follows: NT-shRNA, 5'-CAATGATGGAGA CGGCGCA-3'; S100B-shRNA, 5'-CTGCCACGAGTTCTT TGAA-3'; and shp53, 5'-GACTCCAGTGGTAATCTAC-3'. The lentivirus transfection was performed according to the manufacturer's protocol. Briefly, a total of $1 \times 10^{4}$ OCSCs was treated to obtain single cells and seeded into 24-well plates with $100 \mu \mathrm{l}$ culture medium. Then, $2 \times 10^{5} \mathrm{IU}$ of lentivirus and $0.5 \mu$ l Polybrene (Shanghai GeneChem Co., Ltd.) were added into the aforementioned 24-well plates, and the multiplicity of infection (MOI) value was 20 . After $12 \mathrm{~h}$, the medium containing the lentivirus was replaced with normal medium.

Flow cytometry. OCSCs transfected with shNT or shS100B were treated with cisplatin $(10 \mu \mathrm{M})$ for $48 \mathrm{~h}$. For the treatment of OCSCs with pifithrin- $\alpha$ (a p53 reversible inhibitor; Calbiochem; EMD Millipore, Billerica, MA, USA), the cells were treated with $20 \mu \mathrm{M}$ pifithrin- $\alpha 4 \mathrm{~h}$ prior to cisplatin exposure. In addition, the cells were treated to obtain single cells and incubated with PE-conjugated Annexin V antibody and PI (1:500; 556570; BD Biosciences, Franklin Lakes, NJ, USA) at room temperature for $15 \mathrm{~min}$. The cells were washed with phosphate-buffered saline (PBS) twice and determined by a flow cytometer (BD Biosciences). The data were analyzed by FlowJo software, and the Annexin $\mathrm{V}^{+} \mathrm{PI}^{+}$OCSCs were recognized as 'apoptotic cells', and the Annexin V'PI' OCSCs as 'live cells'.

Statistical analysis. All quantitative data were expressed as the mean \pm SEM. The independent samples t-test, ANOVA, Tukey's multiple comparison test and Pearson's correlation analysis were used for the statistical analysis. Differences were considered statistically significant when $P<0.05$. All statistical analyses were carried out with SPSS 18.0 software (SPSS, Inc., Chicago, IL, USA).

\section{Results}

OCSCs are more resistant to cisplatin than non-OCSCs. In our previous study, we isolated CD133+ OCSCs from A2780 cells and primary tumor tissues, which were demonstrated to harbor self-renewal properties and tumorigenicity (18). In the present study, we performed a CCK- 8 assay to further examine the chemoresistance ability of $\mathrm{CD} 133^{+}$OCSCs and CD133- non-OCSCs, which were obtained from A2780 cells and primary tumor tissues as previously described (13). After $48 \mathrm{~h}$ of treatment with different concentrations of cisplatin, the cell viability of OCSCs was significantly higher than that of non-OCSCs (Fig. 1A and B). Therefore, OCSCs were more resistant to cisplatin than were non-OCSCs.

S100B expression is upregulated in OCSCs and cisplatin-resistant patient samples. Herein, we first verified the expression of S100B in OCSCs. Immunofluorescence staining revealed that S100B expression was higher in A2780-derived OCSCs than in non-OCSCs A2780 cells (Fig. 2A). In addition, S100B mRNA expression was much higher in OCSCs when compared with that in non-OCSCs (Fig. 2B). Moreover, S100B expression was also found to be 3-5-fold higher in A2780- and patient-derived OCSCs at the protein level (Fig. 2C). These findings revealed that $\mathrm{S} 100 \mathrm{~B}$ expression is higher in OCSCs. To further explore the relationship between S100B expression 
A

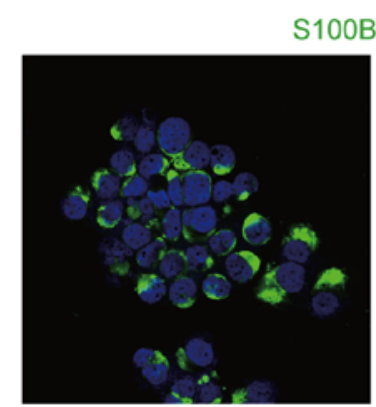

ocsCs

C

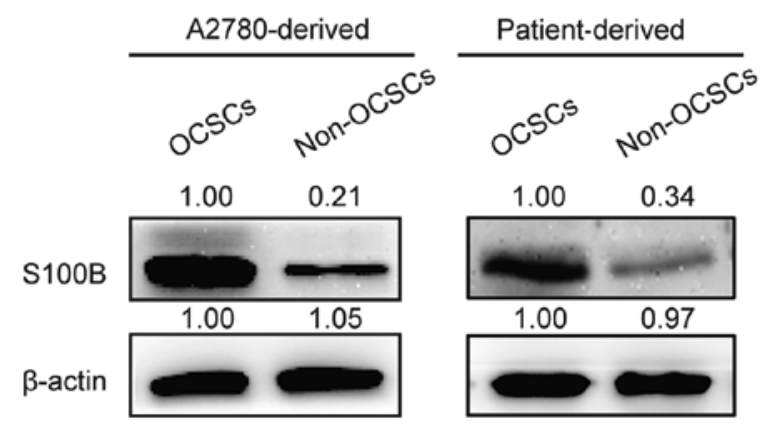

B

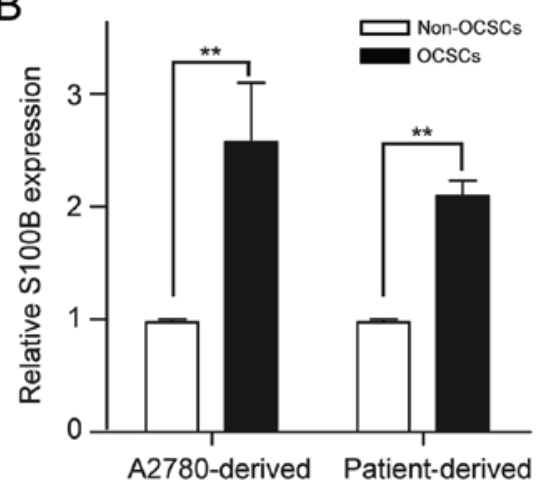

D

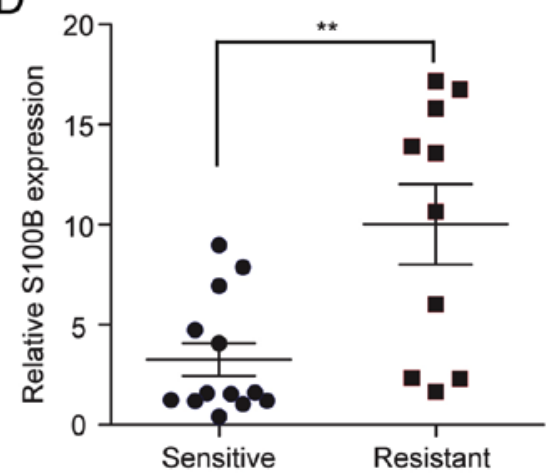

Figure 2. S100B expression is upregulated in OCSCs and cisplatin-resistant patient samples. The expression of S100B was examined by (A) immunofluorescence (bar, $100 \mu \mathrm{m}$ ) (B) real-time PCR and (C) western blotting in A2780- and patient-derived OCSCs and non-OCSCs. (D) S100B expression in cisplatin-sensitive and cisplatin-resistant ovarian cancer patients was analyzed. Representative data from three independent experiments are shown. ${ }^{* *} \mathrm{P}<0.01$. OCSCs, ovarian cancer stem cells.

and chemoresistance in ovarian cancer patients, we analyzed S100B expression in a cohort containing 13 cisplatin-sensitive ovarian cancer samples and 10 cisplatin-resistant samples (GSE51373, high-grade serous ovarian cancer, III-IV stage). We found that S100B expression was significantly higher in cisplatin-resistant patients than in cisplatin-sensitive patients (Fig. 2D), which implied that S100B may be involved in chemoresistance to cisplatin in ovarian cancer patients.

S100B knockdown attenuates cisplatin-resistance in OCSCs. To further investigate the role of S100B in OCSC resistance to cisplatin, we knocked down S100B in A2780and patient-derived OCSCs by transfecting them with S100B shRNA. We found that S100B was clearly knocked down at both the mRNA and protein levels after S100B shRNA transfection (Fig. 3A and B). A CCK-8 assay revealed that S100B silencing led to a significant reduction of cell viability in A2780- and patient-derived OCSCs after treatment with different concentrations of cisplatin for $48 \mathrm{~h}$ (Fig. 3C and E). To further examine the effects of S100B knockdown on OCSC chemoresistance, flow cytometry was performed to determine the apoptosis of OCSCs after treatment with cisplatin. We found that the apoptosis of A2780-derived OCSCs transfected with shNT was not affected after treatment with cisplatin. However, S100B knockdown significantly increased the apoptosis of OCSCs induced by cisplatin (Fig. 3D). Moreover, the same results were obtained in patient-derived OCSCs when S100B was knocked down (Fig. 3F). These data revealed that
S100B knockdown attenuated the chemoresistance of OCSCs to cisplatin.

S100B promotes OCSC chemoresistance by regulating p53. It has been reported that $\mathrm{S} 100 \mathrm{~B}$ can inhibit p53 activation in many cancers. Moreover, p53 is a well-known tumor suppressor that can induce cell apoptosis. Thus, we speculated that S100B may promote OCSC chemoresistance through inhibition of p53. Clearly, S100B knockdown led to elevated expression of p53 and phosphorylated-p53 (p-p53) (Fig. 4A and B). In addition, S100B knockdown also significantly elevated the protein levels of two of p53 downstream effector genes, p21 and MDM2 (mouse double minute 2 homolog gene) (9), which further ascertained the activation of p53 (Fig. 4A and B). Furthermore, p53 knockdown could partially reverse the decreased chemoresistance caused by S100B silencing in A2780-derived OCSCs (Fig. 4C). In addition, we found that the increased apoptosis of OCSCs that was induced by S100B knockdown was also significantly reversed in patient-derived OCSCs when p53 was inhibited (Fig. 4D and E). These results indicated that the effect of S100B on OCSC chemoresistance may be mediated by a p53-dependent pathway.

$S 100 B$ promotes the expression of MDRl and MRPl in $O C S C s$. Chemoresistance in various types of cancers has been demonstrated to be associated with the overexpression of multidrug resistance gene-1 (MDR1) and MDR-associated 
A

A2780-derived

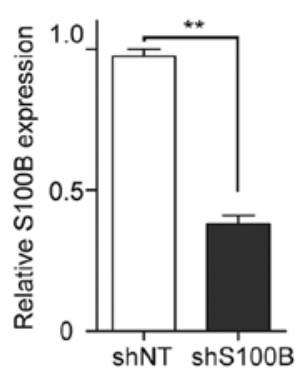

Patient-derived

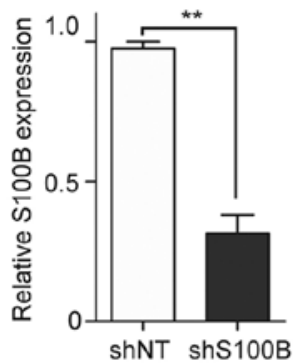

B

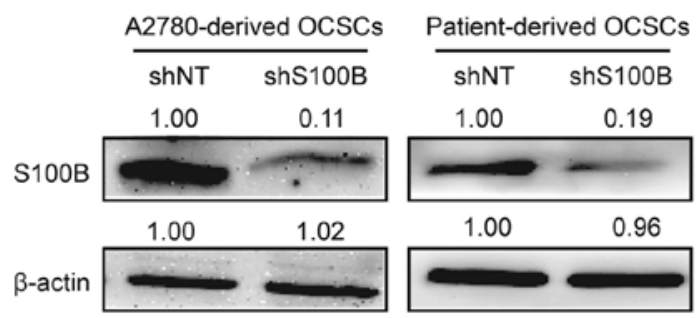

C

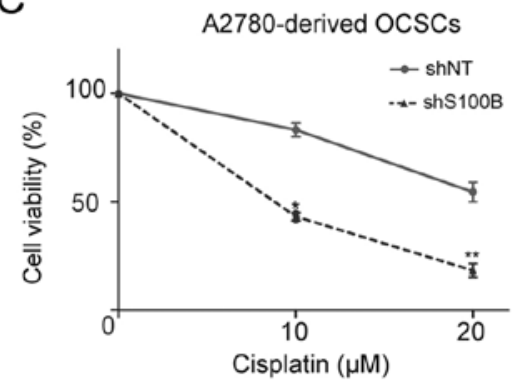

E

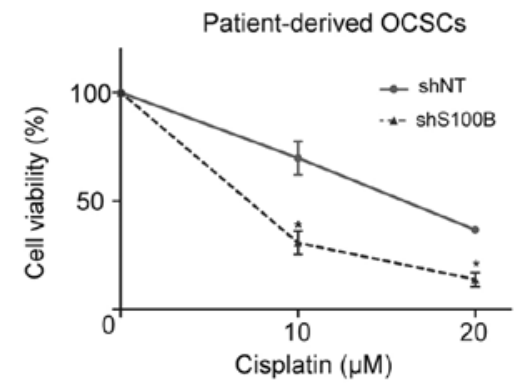

D
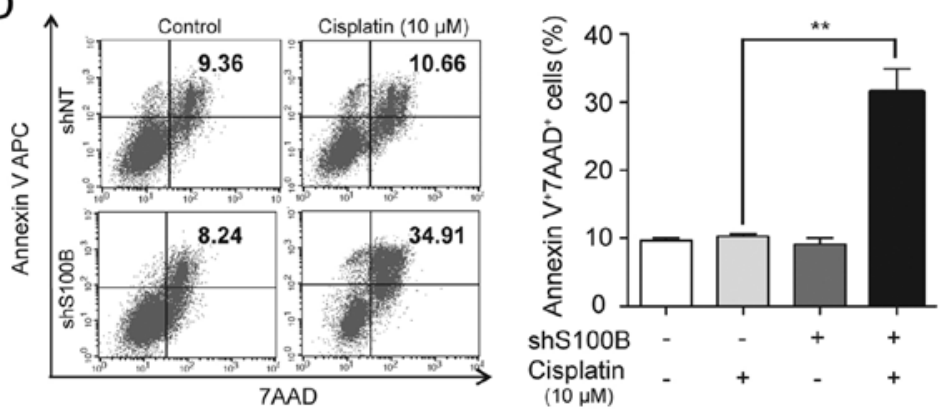

$\mathrm{F}$

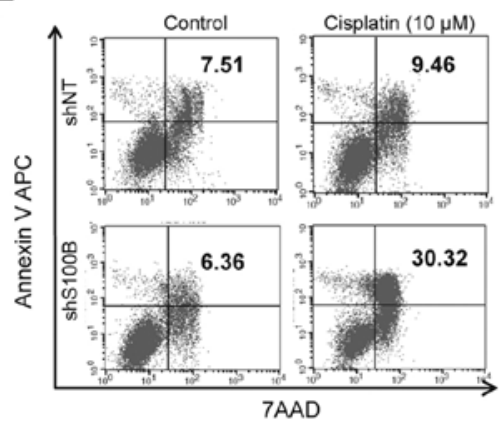

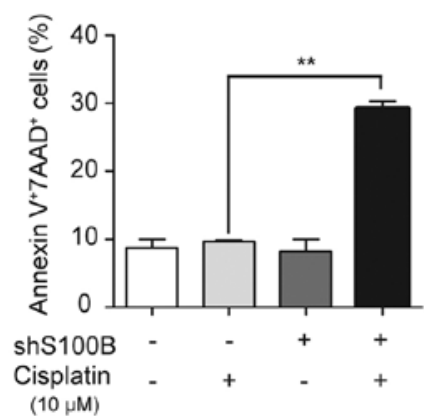

Figure 3. S100B knockdown attenuates cisplatin-resistance in OCSCs. (A) S100B mRNA was examined by real-time PCR in OCSCs transfected with the shS100B vector. (B) S100B protein was examined by western blotting in OCSCs transfected with the shS100B vector. (C and E) A2780- and patient-derived OCSCs with or without S100B knockdown were treated with different concentrations of cisplatin, and their viability was assessed by a CCK-8 assay. (D and F) The apoptosis of A2780- and patient-derived OCSCs with or without S100B knockdown was assessed by flow cytometry after treatment with different concentrations of cisplatin. Representative data from three independent experiments are shown. ${ }^{*} \mathrm{P}<0.05$, ${ }^{* *} \mathrm{P}<0.01$. shNT, non-targeting shRNA; shS100B, S100B shRNA; OCSCs, ovarian cancer stem cells.

protein-1 (MRP1). In addition, p53 regulated MDR1 and MRP1 by modulating the activity of their promoters. Thus, we hypothesized that S100B promotes OCSC chemoresistance by regulating MDR1 and MRP1 expression via p53. To confirm our hypothesis, we first examined the expression of these two genes in patient-derived OCSCs when S100B was knocked down. We found that S100B silencing led to significantly decreased expression of MDR1 and MRP1 (Fig. 5A). Moreover, p53 knockdown partially rescued the decreases induced by S100B silencing in A2780-derived OCSCs (Fig. 5B). These data indicated that S100B may promote chemoresistance by indirectly regulating MDR1 and MRP1 expression.

To further study the influence of S100B on MDR1/MRP1 expression, we analyzed the correlations of S100B and
MDR1/MRP1 expression in a cohort consisting of 157 ovarian cancer patients from the GEO database (GSE13876). Notably, S100B expression was positively correlated with MDR1 and MRP1 expression (Fig. 5C). These results further confirmed that S100B promoted MDR1 and MRP1 expression in ovarian cancer.

\section{Discussion}

Chemotherapy failure is the main cause of recurrence and poor prognosis in ovarian cancer patients (19). Extracellular vesicle (EV)-transferring miRNAs (miR-506, miR-433 and others) (20,21), CAF-producing glutathione (GSH) (22) and immune inhibitory cell-secreted inflammatory factors (23) 
A

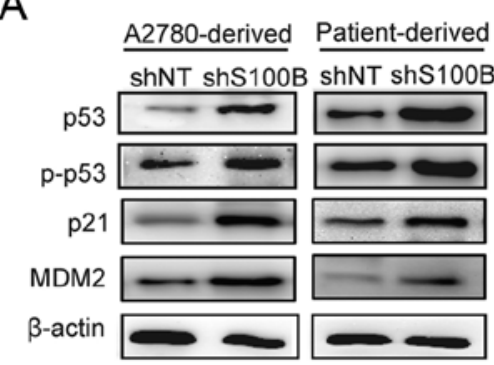

B

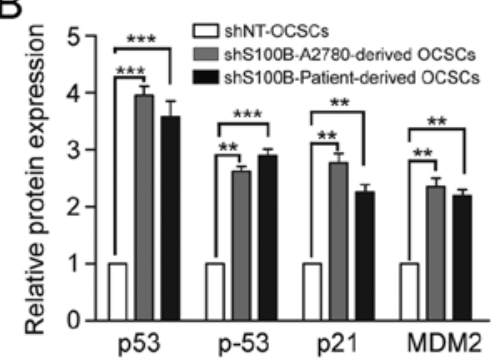

C

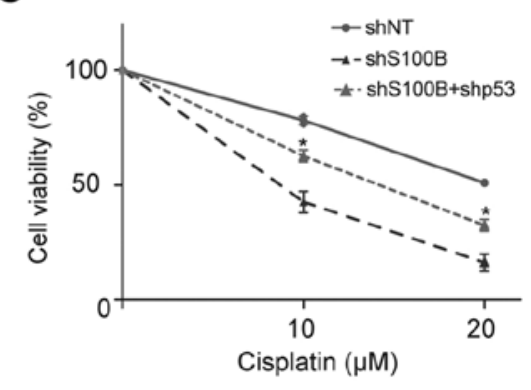

D

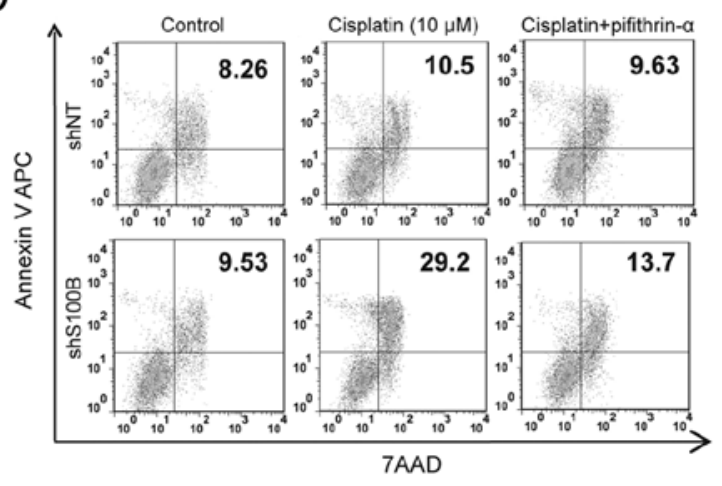

E

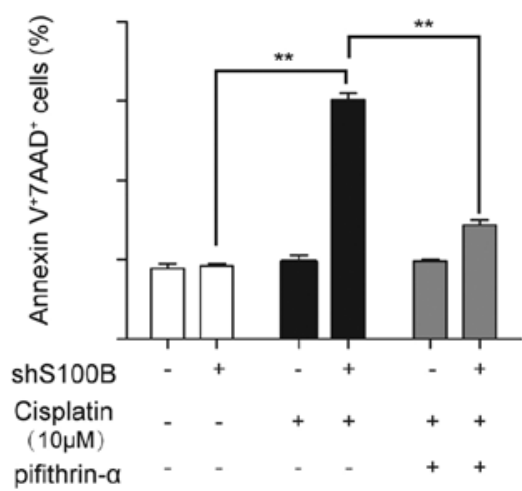

Figure 4. S100B promotes OCSC chemoresistance by regulating p53. (A) The expression of p53, phosphorylation of p53 (p-p53), p21 and MDM2 were detected by western blotting in A2780-(left panel) and patient-derived OCSCs (right panel) transfected with vector or shS100B. (B) The quantitative results of p53, p-p53, p21 and MDM2 expression in shNT- and shS100B-transfected OCSCs. (C) A2780-derived OCSCs transfected with shS100B or shp53 were treated with different concentrations of cisplatin, and their viability was assessed by a CCK-8 assay. (D) The apoptosis of patient-derived OCSCs transfected with shS100B was assessed by flow cytometry after treatment with cisplatin and/or pifithrin- $\alpha$ (a p53 reversible inhibitor). (E) The percentage of apoptotic cells is revealed in patient-derived OCSCs transfected with shS100B after treatment with cisplatin and/or pifithrin- $\alpha$. Representative data from three independent experiments are shown. ${ }^{*} \mathrm{P}<0.05,{ }^{* *} \mathrm{P}<0.01,{ }^{* * *} \mathrm{P}<0.001$. shNT, non-targeting shRNA; shS100B, S100B shRNA; shp53, p53 shRNA; p-p53, phosphorylation of p53; MDM2, mouse double minute 2 homolog gene; OCSCs, ovarian cancer stem cells.

have been reported to activate a series of signaling pathways, including STAT3 (24), Akt (25) and NF- $\kappa \mathrm{B}(26,27)$, to mediate the chemoresistance of ovarian cancer cells. Over the past decade, the failure of cancer therapy has also been attributed to the persistence of cancer stem cells $(28,29)$. However, the molecular mechanisms of drug-resistance in OCSCs remain unclear. In our study, we found that $\mathrm{S} 100 \mathrm{~B}$ was overexpressed in OCSCs and mediated their cisplatin-resistance, which was dependent on p53 inactivation.

The overexpression of $\mathrm{S} 100 \mathrm{~B}$ has been reported in various cancers, such as melanoma, colorectal cancer and non-small-cell lung carcinoma (NSCLC) $(5,30)$. Moreover, S100B levels have been demonstrated to be positively correlated with relapse, distant metastasis or chemoimmunotherapy resistance in these cancers (31). In ovarian cancer, S100B expression was significantly higher in recurrent cancer than in primary cancer (32). Furthermore, S100B expression was found to be upregulated in OCSCs, which could mediate their self-renewal in our previous study (8). In this study, we further found that S100B promoted cisplatin resistance in OCSCs, which may partially explain the previous finding that $\mathrm{S} 100 \mathrm{~B}$ levels were higher in recurrent ovarian cancer. However, whether S100B overexpression potentially regulates CSCs in melanoma, colorectal cancer and NSCLCs remains to be explored.
S100B exerts its functions through extracellular or intracellular pathways. Extracellular S100B can bind RAGE (receptor for advanced glycation end products) to activate the $\mathrm{NF}-\kappa \mathrm{B}$ signaling pathway (33), and intracellular S100B can inhibit p53 to affect cell proliferation or apoptosis (9). We previously found that RAGE was rarely expressed in OCSCs, which eliminated the possibility that S100B regulates OCSC chemoresistance by an extracellular pathway. Fortunately, we found increased p53 and p-p53 expression in OCSCs after knocking down S100B. Moreover, p53 silencing partially rescued the decreased resistance to cisplatin of OCSCs caused by S100B knockdown. These findings indicated that an intracellular, rather than extracellular, signal of S100B mediates the chemoresistance of OCSCs.

As a famous tumor suppressor, p53 plays an important role in chemoresistance by regulating the proliferation and apoptosis of cancer cells $(34,35)$. p53 status can predict chemotherapy response and prognosis in various types of cancers (11,36-38). The interaction of S100B and p53 has been demonstrated. The S100B protein can bind the p53 oligomerization domain and the extreme $\mathrm{C}$ terminus of the tumor suppressor p53 to inhibit p53 tetramer formation and phosphorylation (9). In melanoma, S100B silencing can sensitize cancer cells to UV radiation by inhibiting p53 (6), which may support our findings that S100B 
A

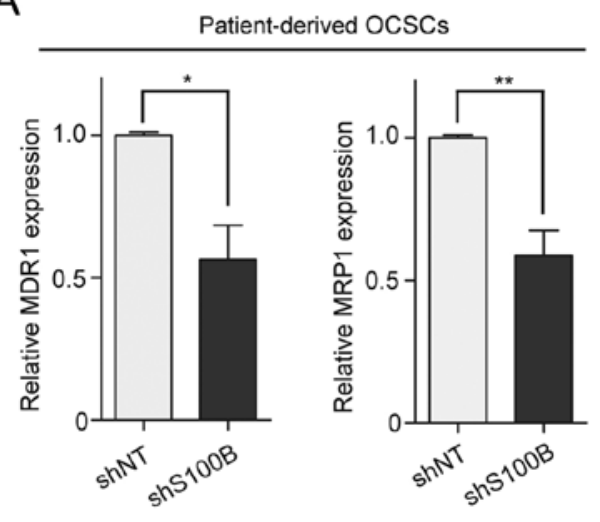

B

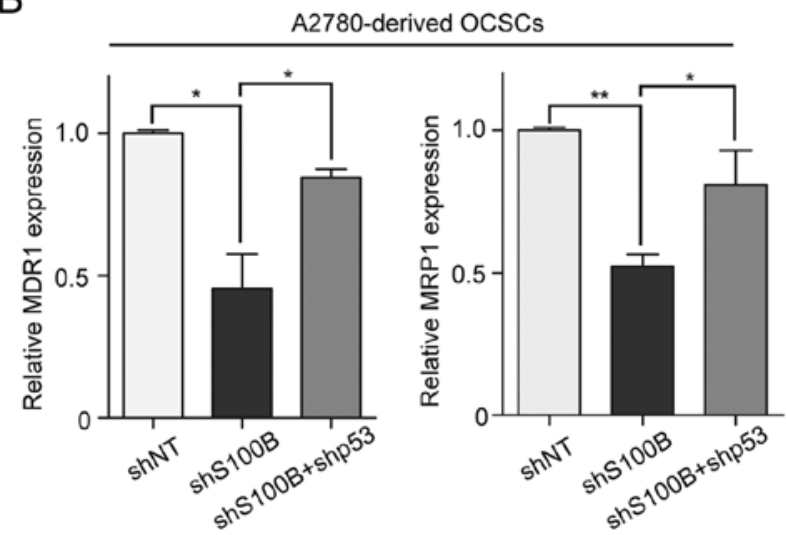

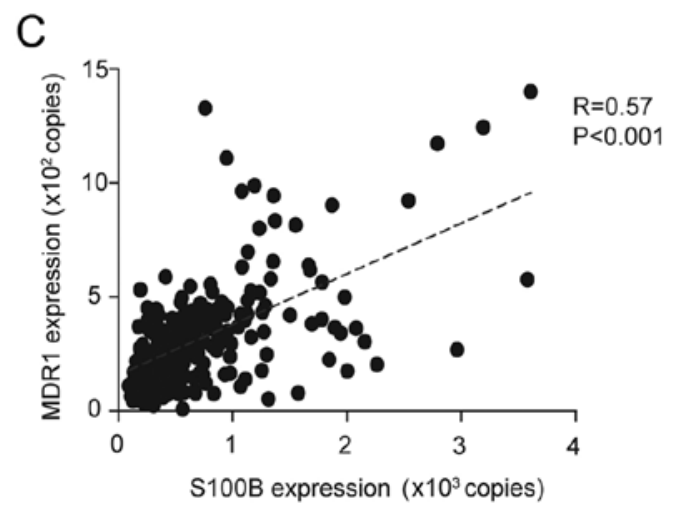

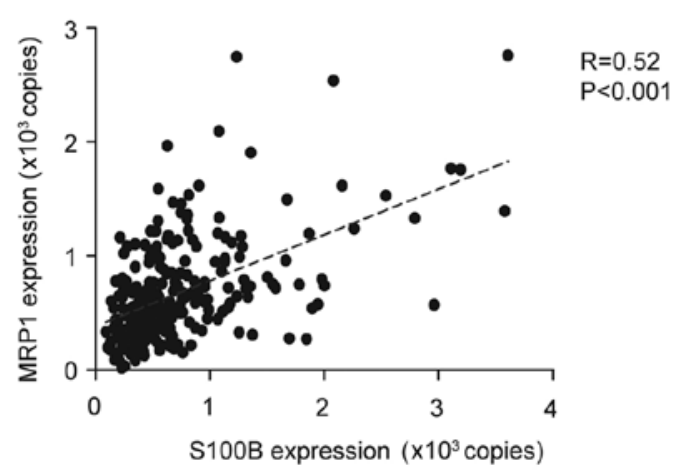

Figure 5. S100B promotes the expression of MDR1 and MRP1 in OCSCs. (A and B) The mRNA expression of MDR1 and MRP1 was detected by western blotting in A2780- and patient-derived OCSCs transfected with vector, shS100B or shp53. (C) The correlations between S100B and MDR1 or MRP1 were analyzed in a cohort from the GEO database. Representative data from three independent experiments are shown. ${ }^{*} \mathrm{P}<0.05$, ${ }^{* *} \mathrm{P}<0.01$. MDR 1 , multidrug resistance gene 1 ; MRP1, multidrug resistance-associated protein 1; OCSCs, ovarian cancer stem cells.

silencing could sensitize OCSCs to cisplatin treatment by the same mechanism. Furthermore, p53 was reported to promote the selective resistance of tumor cells to chemotherapeutic agents by MDR1 (39) and MRP1 (40) gene expression, which was also determined in our study.

It is known that ovarian cancer is a highly heterogeneous disease with different histological subtypes, in which epithelial ovarian cancer (EOC) accounts for $>90 \%$. It has been demonstrated that different histological subtypes of EOC exhibit different genetic profiles, which suggests that 95\% of high-grade serous ovarian cancer (HGSOC) harbor mutant p53 status, while others (including low-grade serous, endometrioid, clear cell and mucinous ovarian carcinomas) harbor wild-type p53 (41). Therefore, besides S100B-p53 interactions, there may be other mechanisms involved in the regulation of OCSC chemoresistance by S100B. As shown in Fig. 2D, S100B expression was higher in cisplatin-resistant HGSOC patients than that in cisplatin-sensitive ones which may also corroborate this hypothesis. In addition, our findings that p53 knockdown could only partially rescue the chemoresistant property of OCSCs, may be additional evidence of the existence of other mechanisms. A recent study reported that S100B-RSK involvement was a novel molecular mechanism in melanoma (42). However, whether the S100B-RSK involvement exists in OCSC chemoresistance regulation, was not explored and is a limitation of our present study.
In conclusion, our study demonstrated that the overexpression of S100B decreased the sensitivity of OCSCs to cisplatin through the inactivation of $\mathrm{p} 53$. These findings provide important evidence that blocking S100B may be a viable means of overcoming the chemoresistance of ovarian cancer. Moreover, numerous small-molecule inhibitors targeting the S100B-p53 interaction have been investigated in cancer treatments (43), which may be combined with chemotherapy for ovarian cancer treatment in the future.

\section{Acknowledgements}

The authors are grateful for the support of our group at the Oncology Department of the Hainan Branch of PLA General Hospital.

\section{Funding}

No funding was received.

\section{Availability of data and materials}

All data generated or analyzed during this study are included in this published article. The datasets used and/or analyzed during the current study are available from the corresponding author upon reasonable request. 


\section{Authors' contributions}

HL and FL conceived and designed the study. TY and JC developed the methodology. FL and JY acquired the data (provided managed patients and facilities). TY, JC and BY analyzed and interpreted the data (e.g., statistical analysis, biostatistics, computational analysis). HL and TY wrote, reviewed, and/or revised the manuscript. JY and TY were responsible for administrative, technical, or material support (i.e., reporting or organizing data, constructing databases). FL and JC supervised the study. All authors read and approved the manuscript and agree to be accountable for all aspects of the research in ensuring that the accuracy or integrity of any part of the work are appropriately investigated and resolved.

\section{Ethics approval and consent to participate}

The study was approved by the Institutional Review Board of the Hainan Branch of Chinese PLA General Hospital (Hainan, China) and informed consent was obtained from all patients.

\section{Patient consent for publication}

Not applicable.

\section{Competing interest}

The authors declare that they have no competing interests.

\section{References}

1. Jayson GC, Kohn EC, Kitchener HC and Ledermann JM: Ovarian cancer. Lancet 384: 1376-1388, 2014.

2. Chiara S, Conte P, Franzone P, Orsatti M, Bruzzone $M$, Rubagotti A, Odicino F, Rugiati S, Carnino F and Rosso R High-risk early-stage ovarian cancer. Randomized clinical trial comparing cisplatin plus cyclophosphamide versus whole abdominal radiotherapy. Am J Clin Oncol 17: 72-76, 1994.

3. Hu L, McArthur C and Jaffe RB: Ovarian cancer stem-like side-population cells are tumourigenic and chemoresistant. Br J Cancer 102: 1276-1283, 2010.

4. Steg AD, Bevis KS, Katre AA, Ziebarth A, Dobbin ZC, Alvarez RD, Zhang K, Conner M and Landen CN: Stem cell pathways contribute to clinical chemoresistance in ovarian cancer. Clin Cancer Res 18: 869-881, 2012.

5. Huang MY, Wang HM, Chang HJ, Hsiao CP, Wang JY and Lin SR: Overexpression of S100B, TM4SF4, and OLFM4 genes is correlated with liver metastasis in Taiwanese colorectal cancer patients. DNA Cell Biol 31: 43-49, 2012.

6. Lin J, Yang Q, Wilder PT, Carrier F and Weber DJ: The calcium-binding protein S100B down-regulates p53 and apoptosis in malignant melanoma. J Biol Chem 285: 27487-27498, 2010.

7. Hamberg AP, Korse CM, Bonfrer JM and de Gast GC: Serum $\mathrm{S} 100 \mathrm{~B}$ is suitable for prediction and monitoring of response to chemoimmunotherapy in metastatic malignant melanoma. Melanoma Res 13: 45-49, 2003.

8. Yang T, Cheng J, Yang Y, Qi W, Zhao Y, Long H, Xie R and Zhu B: S100B mediates stemness of ovarian cancer stem-like cells through inhibiting p53. Stem Cells 35: 325-336, 2017.

9. Lin J, Blake M, Tang C, Zimmer D, Rustandi RR, Weber DJ and Carrier F: Inhibition of p53 transcriptional activity by the S100B calcium-binding protein. J Biol Chem 276: 35037-35041, 2001.

10. Lin J, Yang O, Yan Z, Markowitz J, Wilder PT, Carrier F and Weber DJ: Inhibiting S100B restores p53 levels in primary malignant melanoma cancer cells. J Biol Chem 279: 34071-34077, 2004.

11. Buttitta F, Marchetti A, Gadducci A, Pellegrini S, Morganti M, Carnicelli V, Cosio S, Gagetti O, Genazzani AR and Bevilacqua G: p53 alterations are predictive of chemoresistance and aggressiveness in ovarian carcinomas: A molecular and immunohistochemical study. Br J Cancer 75: 230-235, 1997.
12. Fraser M, Leung BM, Yan X, Dan HC, Cheng JQ and Tsang BK: p53 is a determinant of X-linked inhibitor of apoptosis protein/Akt-mediated chemoresistance in human ovarian cancer cells. Cancer Res 63: 7081-7088, 2003.

13. Xiang T, Long H, He L, Han X, Lin K, Liang Z, Zhuo W, Xie R and Zhu B: Interleukin-17 produced by tumor microenvironment promotes self-renewal of $\mathrm{CD}_{133^{+}}$cancer stem-like cells in ovarian cancer. Oncogene 34: 165-176, 2015.

14. Koti M, Gooding RJ, Nuin P, Haslehurst A, Crane C, Weberpals J, Childs T, Bryson P, Dharsee M, Evans K, et al: Identification of the IGF1/PI3K/NFKB/ERK gene signalling networks associated with chemotherapy resistance and treatment response in high-grade serous epithelial ovarian cancer. BMC Cancer 13: 549,2013

15. Crijns AP, Fehrmann RS, de Jong S, Gerbens F, Meersma GJ, Klip HG, Hollema H, Hofstra RM, te Meerman GJ, de Vries EG and van der Zee AG: Survival-related profile, pathways, and transcription factors in ovarian cancer. PLoS Med 6: e24, 2009.

16. Konstantinopoulos PA, Spentzos D, Karlan BY, Taniguchi T, Fountzilas E, Francoeur N, Levine DA and Cannistra SA: Gene expression profile of BRCAness that correlates with responsiveness to chemotherapy and with outcome in patients with epithelial ovarian cancer. J Clin Oncol 28: 3555-3561, 2010.

17. Livak KJ and Schmittgen TD: Analysis of relative gene expression data using real-time quantitative PCR and the 2(-Delta Delta C(T)) method. Methods 25: 402-408, 2001.

18. Long H, Xie R, Xiang T, Zhao Z, Lin S, Liang Z, Chen Z and Zhu B: Autocrine CCL5 signaling promotes invasion and migration of $\mathrm{CD}_{133^{+}}$ovarian cancer stem-like cells via NF- $\mathrm{KB}$-mediated MMP-9 upregulation. Stem Cells 30: 2309-2319, 2012.

19. Vaughan S, Coward JI, Bast RC Jr, Berchuck A, Berek JS, Brenton JD, Coukos G, Crum CC, Drapkin R, Etemadmoghadam D, et al: Rethinking ovarian cancer: Recommendations for improving outcomes. Nat Rev Cancer 11: 719-725, 2011.

20. Konecny GE, Wang C, Hamidi H, Winterhoff B, Kalli KR, Dering J, Ginther C, Chen HW, Dowdy S, Cliby W, et al: Prognostic and therapeutic relevance of molecular subtypes in high-grade serous ovarian cancer. J Natl Cancer Inst 106: 249, 2014.

21. Weiner-Gorzel K, Dempsey E, Milewska M, McGoldrick A, Toh V, Walsh A, Lindsay S, Gubbins L, Cannon A, Sharpe D, et al: Overexpression of the microRNA miR-433 promotes resistance to paclitaxel through the induction of cellular senescence in ovarian cancer cells. Cancer Med 4: 745-758, 2015.

22. Wang W, Kryczek I, Dostál L, Lin H, Tan L, Zhao L, Lu F, Wei S, Maj T, Peng D, et al: Effector t cells abrogate stroma-mediated chemoresistance in ovarian Cancer Cell 165: 1092-1105, 2016.

23. Reinartz S, Finkernagel F, Adhikary T, Rohnalter V, Schumann T, Schober Y, Nockher WA, Nist A, Stiewe T, Jansen JM et al: A transcriptome-based global map of signaling pathways in the ovarian cancer microenvironment associated with clinical outcome. Genome Biol 17: 108, 2016.

24. Yu H, Lee H, Herrmann A, Buettner R and Jove R: Revisiting STAT3 signalling in cancer: New and unexpected biological functions. Nat Rev Cancer 14: 736-746, 2014.

25. Guo RX, Qiao YH,Zhou Y,Li LX, Shi HR and Chen KS: Increased staining for phosphorylated AKT and nuclear factor-kappaB p65 and their relationship with prognosis in epithelial ovarian cancer. Pathol Int 58: 749-756, 2008.

26. Darb-Esfahani S, Sinn BV, Weichert W, Budczies J, Lehmann A, Noske A, Buckendahl AC, Muller BM, Sehouli J, Koensgen D, et al: Expression of classical NF-kappaB pathway effectors in human ovarian carcinoma. Histopathology 56: 727-739, 2010.

27. Godwin P, Baird AM, Heavey S, Barr MP, O'Byrne KJ and Gately K: Targeting nuclear factor-kappa B to overcome resistance to chemotherapy. Front Oncol 3: 120, 2013.

28. Abdullah LN and Chow EK: Mechanisms of chemoresistance in cancer stem cells. Clin Transl Med 2: 3, 2013.

29. Alvero AB, Chen R, Fu HH, Montagna M, Schwartz PE, Rutherford T, Silasi DA, Steffensen KD, Waldstrom M, Visintin I and Mor G: Molecular phenotyping of human ovarian cancer stem cells unravels the mechanisms for repair and chemoresistance.Cell Cycle 8: 158-166, 2009.

30. Jiang W, Jia Q, Liu L, Zhao X, Tan A, Ma N and Zhang H: S100B promotes the proliferation, migration and invasion of specific brain metastatic lung adenocarcinoma cell line. Cell Biochem Funct 29: 582-588, 2011. 
31. Hwang CC, Chai HT, Chen HW, Tsai HL, Lu CY, Yu FJ, Huang MY and Wang JY: S100B protein expressions as an independent predictor of early relapse in UICC stages II and III colon cancer patients after curative resection. Ann Surg Oncol 18: 139-145, 2011.

32. Laios A, O'Toole SA, Flavin R, Martin C, Ring M, Gleeson N, D'Arcy T, McGuinness EP, Sheils O, Sheppard BL and O' Leary JJ: An integrative model for recurrence in ovarian cancer. Mol Cancer 7: 8, 2008

33. Villarreal A, Aviles Reyes RX, Angelo MF, Reines AG and Ramos AJ: S100B alters neuronal survival and dendrite extension via RAGE-mediated NF-kappaB signaling. J Neurochem 117: 321-332, 2011.

34. Bush JA and Li G: Cancer chemoresistance: The relationship between p53 and multidrug transporters. Int J Cancer 98: 323-330, 2002

35. Masciarelli S, Fontemaggi G, Di Agostino S, Donzelli S, Carcarino E, Strano S and Blandino G: Gain-of-function mutant p53 downregulates miR-223 contributing to chemoresistance of cultured tumor cells. Oncogene 33: 1601-1608, 2014.

36. Tsai CM, Chang KT, Wu LH, Chen JY, Gazdar AF, Mitsudomi T, Chen MH and Perng RP: Correlations between intrinsic chemoresistance and HER-2/neu gene expression, p53 gene mutations, and cell proliferation characteristics in non-small cell lung cancer cell lines. Cancer Res 56: 206-209, 1996.

37. Laframboise S, Chapman W, McLaughlin J and Andrulis IL: p53 mutations in epithelial ovarian cancers: Possible role in predicting chemoresistance. Cancer J 6: 302-308, 2000.
38. Kawasaki M, Nakanishi Y, Kuwano K, Yatsunami J, Takayama K and Hara N: The utility of p53 immunostaining of transbronchial biopsy specimens of lung cancer: p53 overexpression predicts poor prognosis and chemoresistance in advanced non-small cell lung cancer. Clin Cancer Res 3: 1195-1200, 1997.

39. Thottassery JV, Zambetti GP, Arimori K, Schuetz EG and Schuetz JD: p53-dependent regulation of MDR1 gene expression causes selective resistance to chemotherapeutic agents. Proc Natl Acad Sci USA 94: 11037-11042, 1997.

40. Bahr O, Wick W and Weller M: Modulation of MDR/MRP by wild-type and mutant p53. J Clin Invest 107: 643-646, 2001.

41. Domcke S, Sinha R, Levine DA, Sander C and Schultz N: Evaluating cell lines as tumour models by comparison of genomic profiles. Nat Commun 4: 2126, 2013.

42. Hartman KG, Vitolo MI, Pierce AD, Fox JM, Shapiro P, Martin SS, Wilder PT and Weber DJ: Complex formation between S100B protein and the p90 ribosomal S6 kinase (RSK) in malignant melanoma is calcium-dependent and inhibits extracellular signal-regulated kinase (ERK)-mediated phosphorylation of RSK. J Biol Chem 289: 12886-12895, 2014.

43. Markowitz J, MacKerell AD Jr and Weber DJ: A search for inhibitors of S100B, a member of the S100 family of calcium-binding proteins. Mini Rev Med Chem 7: 609-616, 2007. 